\title{
Ratchet effect in an underdamped periodic potential and its characterisation
}

\author{
Shantu Saikia1* \\ ${ }^{1}$ St. Anthony's College, Shillong-793001, Meghalaya, India
}

(Dated: August 1, 2018)

\begin{abstract}
Ratchet effect in a driven underdamped periodic potential system is studied. The presence of a space dependent and periodic friction coefficient, but with a phase difference with the symmetric periodic potential is shown to generate substantial ratchet current. The ratchet performance is characterised in terms of the various parameters of transport. The performance of this ratchet is compared with a ratchet with an underlying periodic and asymmetric potential. It is shown that an optimum combination of inhomogeneity in the system and asymmetry of the potential can substantially enhance the performance of an underdamped ratchet.
\end{abstract}

PACS numbers: : 05.40.-a, 05.40. jc, 05.40.Ca

*Electronic address: shantusaikia@anthonys.ac.in 


\section{INTRODUCTION}

Noise or fluctuations are inherent in almost any physical or biological system at a finite temperature. These fluctuations play a dominant role in the system dynamics at the microscopic domain, particularly when their energy scales become comparable to that of the system. Different phenomena have been discovered in which these fluctuations play a counter intuitively constructive role [1- 7$]$.

Ratchet effect [5] is the phenomenon in which directed particle transport takes place in a driven asymmetric periodic potential system without the application of any obvious external bias, aided by noise or fluctuations. The successful application of Ratchet effect in explaining different processes in the Biological and Physical world has led to a spurt of studies - both theoretical and experimental [8 13]. Though primarily studies on Ratchets were aimed at explaining the working of biological or molecular motors [14], presently it has diversified to various other fields [15-18].

For realising ratchet effect, the symmetry of the system has to be broken. Also the system has to be driven away from equilibrium. Based on these conditions different models of Ratchets have been proposed and studied [5].

In this work we study ratchet effect in a model henceforth called as inhomogeneous ratchet. In this ratchet, particle transport occurs in a driven underdamped inhomogeneous periodic potential system. The asymmetry in the system is due to the space dependent friction coefficient which is periodic with the same periodicity as the potential, but with a phase difference with the potential [20-23, 25-33].

Particle transport in inhomogeneous systems driven away from equilibrium has been an active field of research in the past few decades [19 23, 25 38]. In such inhomogeneous systems, the particle experiences a non uniform diffusion. This may be either due to a space dependent friction [20-23, 25-33] or due to a space dependent temperature in the system [34 38].

In nature there are numerous examples of systems having a space dependent friction. For example molecular motor proteins experiences a space dependent friction coefficient when they move along the periodic structures of microtubules [38]. Brownian motion in confined geometries show a space dependent friction [39]. Particles undergoing surface diffusion 
also experiences a space dependent friction coefficient [39, 40]. Space dependent friction coefficient also finds applications in superlattice structures, Josephson Junction equation [41], and ad-atom motion on the surface of a crystal of identical atoms [42].

The various aspects of particle transport in overdamped inhomogeneous systems with a space dependent friction coefficient has been extensively studied [20 24]. It was shown that it is possible to obtain directed particle transport aided by noise even with a perfectly symmetric and periodic potential in the presence of a similarly periodic friction coefficient, but with a phase difference $\phi(\phi \neq 0, \pi)$ with the potential; the system being driven away from equilibrium. The various parameters characterising the transport and particle current, like the particle velocity, efficiency and coherence of transport, diffusion etc. have been studied. Also it has been shown that forced inhomogeneous ratchets exhibit current reversals.

Though overdamped approximation is valid for many physical and biological processes and systems [5] - for example molecular motor movement in the Brownian regime - inertial effects can, however, play an important role in many other situations [25-37, 42 49]. Examples being the diffusion of ad-atoms on a crystal surface [50], dissipation in threshold devices [51], dislocation of defects in metals [52] and Josephson junctions [53].

Particle dynamics in overdamped and underdamped systems are fundamentally different. In the overdamped regime, inertial effects can be ignored. The particle in such a system exhibits a hopping motion between the wells of the potential. The particles gets trapped in a potential minima. With noise, the particles escape from the locked state in a potential well only to be trapped in another adjacent well. On the other hand in the underdamped dynamics, in a periodic potential, the particle can be in two states of dynamics - the locked state in which the particles are trapped in a potential minima and the running state in which because of the particle's momentum the keep travelling over many periods of the potential. In the presence of finite noise the particle undergoes transitions between these two dynamical states [54].

Underdamped dynamics exhibits some interesting phenomena which are otherwise not observed in overdamped systems. For example underdamped systems exhibits a giant enhancement of diffusion coefficient [55]. The diffusion coefficient is dependent on the jump rates out of a potential well and also on the particle jump lengths. These parameters are in turn dependent on the amount of damping. In the underdamped regime, the particle dynamics is dominated by long jumps [56]. 
Inertial inhomogeneous system with a space dependent temperature has been shown to yield directed transport of particles in the absence of any external bias [34 37, 49] .

Particle transport in driven inertial inhomogeneous system with a space dependent friction coefficient has been shown to exhibit other interesting phenomena also [26 33]. Particle current is observed with associated multiple current reversals in the deterministic regime [26]. When driven with a square drive, apart from substantial particle current in optimised parameter regimes, the system also shows dispersionless particle motion at intermediate time regimes [27, 32]. Using this model system the phenomenon of Stochastic Resonance in a periodic potential system was obtained [26-28, 32, 33]. This phenomenon in which a periodically driven system shows an enhanced response to the external drive in the presence of noise or fluctuations, was earlier mostly studied in bi stable systems.

We further explore different characteristics of particle transport in a driven underdamped periodic potential system using the same model as in [26 33]. The performance of this inhomogeneous ratchet is compared with a more commonly studied model in which asymmetry in the system is due to an inherent asymmetry in the underlying periodic potential (henceforth called as asymmetric ratchet) [5, 57 60]. A scheme for obtaining enhanced efficiency of a ratchet in the underdamped regime is proposed by combining essential features of both these ratchets.

For optimising the working of ratchets, their performance needs to be characterised. As Ratchets operate in environments dominated by the random fluctuations, their performances are measured by the fluctuating quantities [61] like position $(x(t))$, velocity $(v)$ or work output $(W)$. In the presence of fluctuations, the particle positions at any given time get spread out when being transported as an ensemble through a distance from a common initial position and time. A measure of this spread is given by the effective diffusion or dispersion $D_{\text {eff }}=\frac{\left\langle x^{2}(t)\right\rangle-\langle x(t)\rangle^{2}}{2 t}$, where the average $\left\langle. .>\right.$ is over an ensemble. But as $D_{\text {eff }}$ does not distinguish between motors of different average velocities, we need a coherency parameter which incorporates both velocity and spread. This is given by the Peclet number [62] $P_{e}=\frac{\langle v\rangle l}{D_{e f f}}$, where $l$ is a characteristic length of the system and $\langle v\rangle$ is the time averaged velocity.

Apart from the Peclet number another important parameter for the characterisation of a Brownian motor is its efficiency. As these motors operate in a viscous environment, they can do work against an external load $F$, and against the viscous drag in the medium or both. 
The efficiency of energy conversion of a motor with respect to the work done against a load

$F[5,63]$ is given as $\eta_{E}=\frac{|F\langle v\rangle|}{P_{i n}}$, where $P_{i n}$ is the input power. Though this definition is thermodynamically correct, it becomes unsatisfactory in the case of Brownian motors which works only via the transport against the viscous drag of the medium, in the absence of a load. A definition of efficiency which takes into account the work done solely against the viscous drag is called the Stokes efficiency, $\eta_{S}=\frac{\langle v\rangle^{2}}{\left\langle v^{2}\right\rangle-T \mid}$ [45]. Suzuki and Munakata [64] combined the above two efficiencies to give a more general definition of efficiency called the rectification efficiency given as $\eta_{r e c}=\frac{\gamma\langle v\rangle^{2}+F\langle v\rangle}{P_{i n}}$.

\section{THE MODEL}

In this work we consider the motion of a particle in a periodic potential $V(x)=$ $-V_{0}(\sin (k x)+b \sin 2 k x)$. The friction coefficient $\gamma(x)=\gamma_{0}(1-\lambda \sin (k x+\phi))$ is periodic with the same periodicity as the potential but has a phase difference $\phi . b$ is the parameter which determines the underlying asymmetry in the potential whereas $\lambda$ is the inhomogeneity parameter. The system is studied under the following approximations.

(i) When the system is inhomogeneous, with a space dependent friction coefficient but with a symmetric potential. For this, $b=0$ and $\lambda \neq 0$. In this case the asymmetry in the system is only due to the inhomogeneity (inhomogeneous ratchet). (ii) When the system is homogeneous but there is an asymmetry in the underlying periodic potential. For this $b \neq 0$ and $\lambda=0$ (asymmetric ratchet) (iii) When the system has both inhomogeneity and asymmetry in the potential. In this case $b$ and $\lambda$ both are $\neq 0$ and the asymmetry in the system is due to both the parameters (combination of inhomogeneous and asymmetric ratchets).

The system is driven periodically by an external periodic forcing $F(t)=F_{0} \cos \left(\omega t+\phi_{0}\right)$. Over a period of the drive, the net external forcing averages out to zero. Hence there is no obvious applied bias in the system. The system is studied in the presence of finite noise.

The one dimensional equation of motion of a particle of mass $m$ is given by the Langevin's equation,

$$
m \frac{d^{2} x}{d t^{2}}=-\gamma(x) \frac{d x}{d t}-\frac{\partial V(x)}{\partial x}+F(t)+\sqrt{\gamma(x) T} \xi(t) .
$$

In Eq. 1 , the temperature $T$ is in units of the Boltzmann constant $k_{B}$. The Gaussian distributed fluctuating forces $\xi(t)$ obeys the statistics: $\left\langle\xi(t)>=0\right.$, and $\left\langle\xi(t) \xi\left(t^{\prime}\right)>=\right.$ 
$2 \delta\left(t-t^{\prime}\right)$. The above equation is written in dimensionless units by setting $m=1, V_{0}=0$ and $k=1$. The dimensionless form of the equation keeping the same symbols for the reduced variables can be written as

$$
\frac{d^{2} x}{d t^{2}}=-\gamma(x) \frac{d x}{d t}+\cos x+2 b \cos 2 x+F(t)+\sqrt{\gamma(x) T} \xi(t)
$$

where $\gamma(x)=\gamma_{0}(1-\lambda \sin (x+\phi))$. In the reduced equation too, the noise variable, $\xi$, satisfies exactly similar statistics as earlier. The potential $V(x)$ and the friction coefficient $\gamma(x)$ are periodic having the same periodicity of $2 \pi . F_{0}$ is amplitude of the external drive and the particles experience a finite barrier between two consecutive wells of the potential $V(x)$ for all values of amplitude $F_{0},\left(0<F_{0}<1\right)$. The barrier disappears momentarily in a period at the critical field value $F_{0}=F_{c}=1$.

For obtaining finite particle current in a driven periodic potential system, the asymmetry of the system needs to be broken. This can be achieved by making the potential asymmetric $(b \neq 0)$ (asymmetric ratchet) or by making the system inhomogeneous $(\lambda \neq 0$, with a value of phase difference $\phi \neq n \pi)$ (inhomogeneous ratchet). In this work, as discussed above we study the system under different approximations.

\section{NUMERICAL RESULTS}

Eq. 2 is solved numerically using the Heun's method [65] for solving stochastic differential equations. The particle trajectories are obtained for given initial conditions for fixed values of the parameters. The steady state mean velocity $\langle\bar{v}\rangle$ of the particle is obtained as

$$
\langle\bar{v}\rangle=\left\langle\lim _{t \rightarrow \infty} \frac{x(t)}{t}\right\rangle,
$$

The system is allowed to evolve over effective times of around $t=10^{5}$ and ensemble averaging $\langle\ldots\rangle$ is done over an ensemble of 50 different initial conditions.

We study particle transport in the model inhomogeneous ratchet. The performance of this ratchet is compared with that of the asymmetric ratchet. Both these ratchets schemes are then combined to evolve a criterion for enhancing the performance of underdamped ratchets. The main results of our work are highlighted below. We also study, the performance of the underdamped ratchets as a function of the other parameters so as to optimise its performance. 


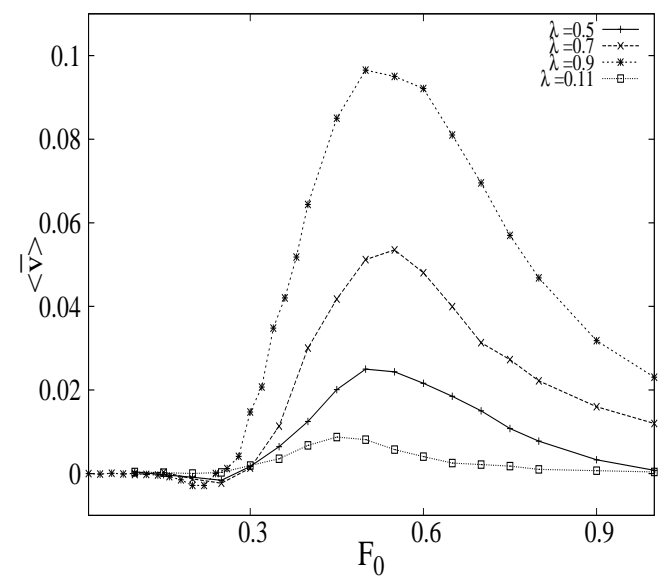

(a)

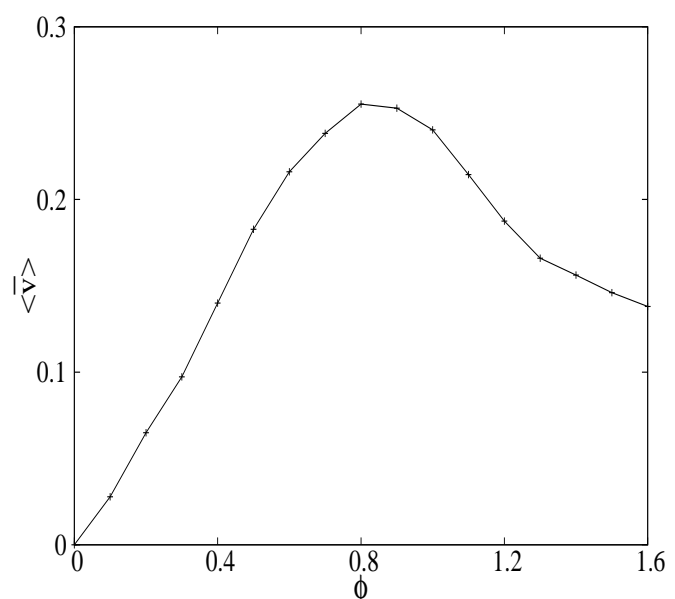

(b)

FIG. 1: Plot of $\langle\bar{v}\rangle$ versus $F_{0}$ for different values of $\lambda$ (a) for $\gamma_{0}=0.12, \tau=1000, \phi=0.35$ and $T=0.4$. Plot (b) shows the variation of $\langle\bar{v}\rangle$ with $\phi$ for $\lambda=0.9, F_{0}=0.5$ and other parameters remaining same as plot (a).

In Fig. 1a the value of average velocity $\langle\bar{v}\rangle$ is plotted as a function of the driving amplitude $F_{0}$ for different values of $\lambda$, keeping the potential symmetric (i.e. $b=0$ ). With a finite value of the parameter $\lambda$ (for a fixed value of $\phi$ ), appreciable ratchet current is obtained. This current is solely due to the asymmetry introduced into the system because of the space dependent friction coefficient. The amount of current in this inhomogeneous ratchet however expectedly depends on the value of the inhomogeneity parameter $\lambda$ (for a fixed value of $\phi$ ).

The nature of variation of the particle current in Fig.1a can be understood by looking at the origin of particle current. The magnitude and direction of particle current - if any in a driven periodic potential depends on the number of interwell particle hoppings to the right and the left of a potential minima. The number of such hops are intricately dependent on the temperature, asymmetry and amplitude of external drive. If there is no asymetry in the system, the hoppings to the right and the left are equally probable giving rise to zero particle current.

In the model inhomogeneous system under our consideration, the asymmetry in the system arises because of the phase difference $\phi$ between the periodic potential and the space dependent friction coefficient. When $\phi$ is equal to $0, \pi$ and $2 \pi$, the friction coefficient about the potential peak is symmetric. However, for $\phi=0.35$ as considered here, the friction coefficient on the left of the potential peaks is larger than on the right. If this system is driven adiabatically [29] or with a finite but small frequency [27, 30], this leads to an asymmetric 
particle distribution about the bottom of the potential well in the dynamic situation, leading to a probability of getting a negative particle current. Also, because of higher friction, the particles will spend more time on the left side of the potential peak, absorbing more heat from the fluctuations leading to higher temperature. This gives rise to a probability of getting a particle current in the positive direction. The final direction of particle current that is obtained is a result of these two competing effects and also is determined by the other parameters of the system.

However, in a recent work [31] it was shown that if the frequency of the external drive is large, at low temperatures the particles spend equal time on either side of the potential peak irrespective of the friction coefficient and the motion remains symmetrical in both directions. However as temperature rises, for finite $\lambda$ difference starts appearing leading to more probability of the particle crossing over to one side from a well than the other.

Therefore in the inhomogeneous system under our consideration, the final magnitude and direction of current is a result of a complex interplay between the non linearity of the system, the particle dynamics, the asymmetry of the system and the choice of the parameters characterising the system.

When the amplitude of the external drive is small, the particles remain near the bottom of the potential wells. So they do not feel the effect of asymmetry that much and they make occasional hoppings out of the well aided by the fluctuations. Hence the particle current is less. As the amplitude increases, the particles can climb further up the potential walls and they experience the asymmetry in thes system more. Also the probability of interwell hoppings aided by fluctuations increases. So the current rises. For very high amplitude though, the barrier to motion is almost disappears it disappears momemtarily in each drive if the amplitude is more than the critical tilt. So the effect of asymmetry is less pronounced leading to less current. In between these two extremes, expectedly the current peaks for an intermediate value of $F_{0}$ (Fig. 1a). The amount of current of course depends on the degree of inhomogeniety in the system which is determined by the parameter $\lambda$. It is observed that as the value of the parameter $\lambda$ increases, the particle current also increases monotonically (Fig.1a).

The degree of asymmetry in this system is intricately linked with the phase difference $\phi$ between the periodic friction coefficient and the underlying periodic potential. Fig. 1b, shows the plot of $\langle\bar{v}\rangle$ as a function of $\phi$. Other parameters remaining fixed, the ratchet 
current is seen to peak for a value of $\phi=0.8$.

This observed variation can be understood by noting that the asymmetry of the system changes with the value of the parameter $\phi$. For $\phi=0, \pi$ and $2 \pi$, the friction coefficient is symmetric about a potential minima leading to zero particle current. For other values of $\phi$ a finite current can be observed as it leads to a difference in the number of particle hoppings to the right and left of a potential minima. Asymmetry in the system is maximum for $\phi=0.5 \pi$. However maximum asymmetry in the system does not necessarily mean the maximum difference in the number of hoppings to the left and the right of a potential well, as this is also dependent on the temperature of the system [31]. Other parameters remaining fixed, current peaks for that value of $\phi$ at which this difference is maximum.

In a recent work [31] a detailed explanation of this has been put forward. The average friction coefficient experienced by the particle and hence its mobility is dependent on $\phi$, which along with the temperature of the system determines the asymmetry in the hoppings.

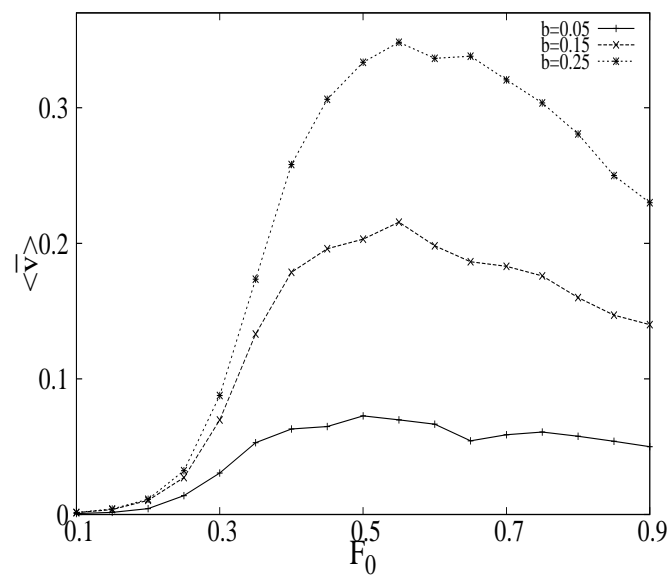

FIG. 2: Plot of $\langle\bar{v}\rangle$ versus $F_{0}$ for different values of $b$, for $\gamma_{0}=0.12, \tau=1000, \phi=0.35$ and $T=0.4$.

Fig. 2 is a plot of the average particle velocity $\langle\bar{v}\rangle$ for the asymmetric ratchet in which the particle experiences a homogeneous friction coefficient $(\lambda=0)$. The asymmetry in the system is due to the asymmetry in the underlying periodic potential. In the figure the average velocity is plotted as a function of the driving amplitude $F_{0}$ for different values of the parameter $b$ which denotes different degrees of asymmetry of the potential. Undoubtedly, higher degrees of asymmetry in the potential gives rise to higher values of particle current.

The observed variation of the particle current with the amplitude for the asymmetric ratchet has the same explanation as that for the inhomogeneous ratchet as given above, 
with the current peaking at an optimal drive amplitude.

Comparison of Fig. 1a and Fig. 2 shows that that in the underdamped regime, an asymmetric ratchet (Fig. 2) gives higher values of particle current than the inhomogeneous ratchet (Fig. 1a) as inhomogeneity is a feeble cause of asymmetry as compared to the inherent asymmetry in the potential itself. However for certain parameter regimes, the inhomogeneous ratchet does give currents higher than that for the asymmetric ratchet. For example current for the inhomogeneous ratchet for $\lambda=0.9$ is higher than that for the asymmetric ratchet for $b=0.05$ Thus, though inhomogeneity is a feeble cause of asymmetry, appreciable ratchet currents can be obtained. As particle transport in such inhomogeneous system finds analogies in various physical and biological systems as mentioned in earlier sections, the inhomogeneous ratchet provides an interesting option for modelling and studying different natural systems.

The drive amplitude at which the particle current peaks will also be dependent on the temperature of the system. For the present case when the drive frequency is very small compared to the natural frequency of the particles at the bottom of a potential minima, in the absence of any fluctuations $(T=0)$, the particles remain near the bottom of the potential minima. No particle current will be observed in this case. However for finite temperature the particle gets kicked around by the fluctuations and they start exploring the asymmetry

of the system. For lower temperatures, if the drive amplitude is small, the probability of the particles overcoming the barrier and hopping to another well is less. However as the amplitude increases the particle moves up further away from the bottom of the well and aided by the fluctuations cross over to another well. The asymmetry in the system will give rise to preferentially more hoppings in a particular direction leading to finite current. However, if the temperature of the system is higher the particles will undergo interwell hoppings at a lesser amplitude of drive. So it can be concluded that the drive amplitude at which the current peaks shifts to higher values for lower temperatures.

\section{CHARACTERISATION OF THE RATCHET CURRENT}

For optimisation of the performance of a ratchet it needs to be characterised in terms of the various parameters which defines the quality of transport. One such parameter is the diffusion coefficient $D_{\text {eff }}$. As the ratchets operate in a very noisy environment which is 
dominated by random fluctuations, the particles in a ratchet spreads during its motion over a period of time. So apart from calculating the average particle velocity, it is necessary to have a measure of the diffusive spread of the particles. The measure of this spread is given by the diffusion coefficient $D_{\text {eff }}=\left(\left\langle x^{2}(t)\right\rangle-\langle x(t)\rangle^{2}\right) / 2 t$, where the averaging $\langle\ldots .$.$\rangle is over$ the ensembles.

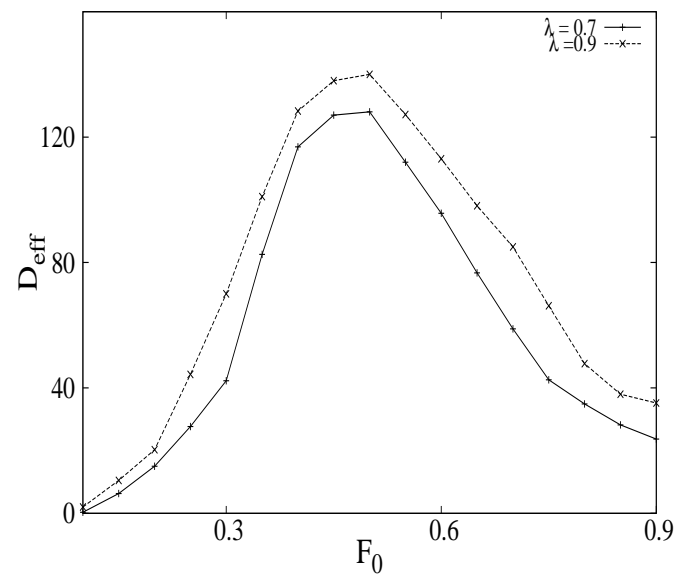

(a)

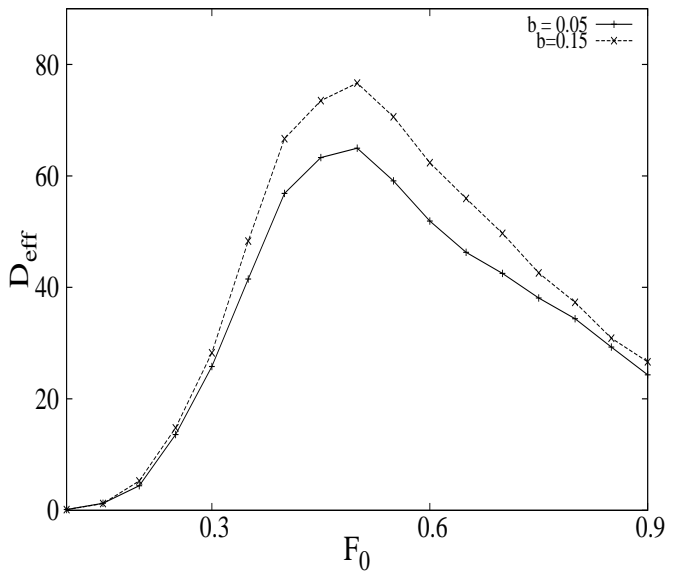

(b)

FIG. 3: Plot of diffusion coefficient $D_{\text {eff }}$ versus $F_{0}$ for different values of $\lambda$ (a) and $b$ (b) for $\gamma_{0}=0.12, \tau=1000, \phi=0.35$ and $T=0.4$.

Fig. $3 \mathrm{a}$ and $3 \mathrm{~b}$ shows the plot of the diffusion coefficient $D_{\text {eff }}$ versus the driving amplitude for the inhomogeneous ratchet and the asymmetric ratchet. Diffusion in both the ratchets peaks around the value of $F_{0}$ at which there is a peak in the average velocity. The degree of spread in the inhomogeneous ratchet is comparatively much higher. This is beacause the degree of asymmetry in the system experienced by the particle leading to its directed motion is higher in the asymmetric ratchet compared to the inhomogeneous ratchet

A measure of the average velocity $\langle\bar{v}\rangle$ or the diffusion coefficient $D_{\text {eff }}$ in isolation will not give the complete picture of the nature of transport in a ratchet. For, a ratchet with high average particle velocity can have high diffusion associated with the transport making the transport incoherent. On the other hand, even transport with lower $D_{\text {eff }}$ does not guarantee higher average velocity. So it is necessary to measure the coherency of transport in a ratchet. A parameter which gives a measure of the coherency of transport and includes both the average velocity $\langle\bar{v}\rangle$ and the diffusion coefficient $D_{\text {eff }}$, is the Peclet number $P_{e}[62]$.

Fig. $4 \mathrm{a}$ and $4 \mathrm{~b}$ shows the plot of Peclet number $\left(P_{e}\right)$ for the inhomogeneous ratchet and the asymmetric ratchet respectively. For both the types of ratchets, Peclet number is much 


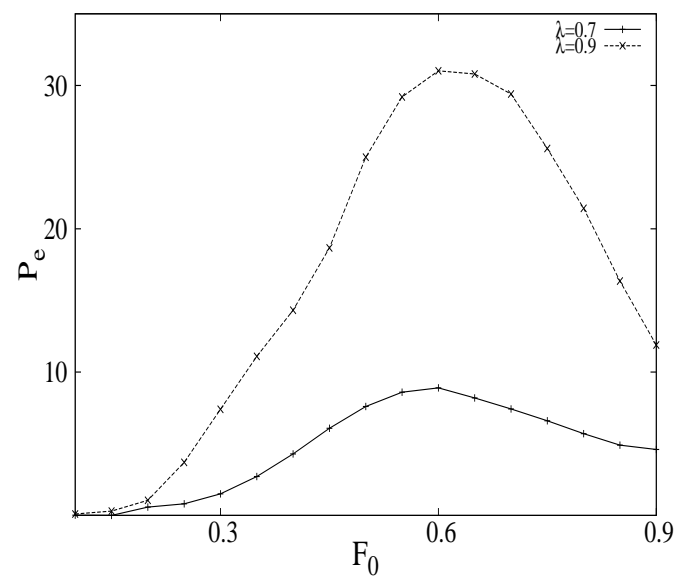

(a)

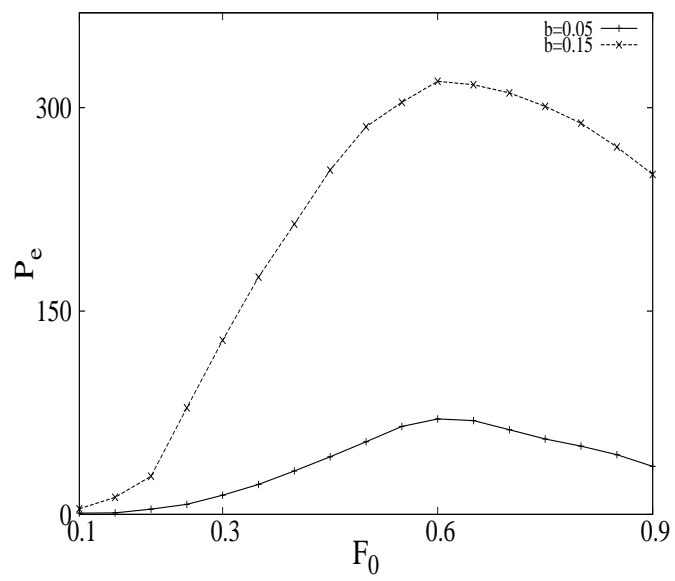

(b)

FIG. 4: Plot of Peclet number $P_{e}$ versus $F_{0}$ for different values of $\lambda$ (a) and $b$ (b) for $\gamma_{0}=0.12$, $\tau=1000, \phi=0.35$ and $T=0.4$.

higher than 2 which proves that though the particle transport is highly diffusive, it is greatly coherent too. However coherency of transport in the asymmetric ratchet is much higher than that of the inhomogeneous ratchet. The coherency of transport is dependent on the degree of asymmetry in the system (i.e. the values of $\lambda$ and $b$ ) in both the types of ratchets.

In the ratchet models under our consideration, though there is no external load, the ratchet does work in transporting particles against the viscous drag of the medium in which it operates. So it is necessary to have a measure of its efficiency. A definition of efficiency which takes into account the work done solely against the viscous drag is called the Stokes efficiency, $\eta_{S}=\frac{\langle v\rangle^{2}}{\left|\left\langle v^{2}\right\rangle-T\right|}[45]$.

Fig. 5 gives a plot of the Stoke's efficiency as a function of the external driving force $F_{0}$ for the two kinds of ratchets under our consideration for the parameters mentioned and for different values of the asymmetry parameter. The efficiency of performance of the ratchets are not only dependent on the degree and type of asymmetry but also on the amplitude of external drive. The efficiency for both the kinds of ratchets peak around the drive amplitude where there is a peaking of the average particle velocity.

We also have studied the inhomogeneous ratchet as a function of the other system parameters. Fig. 6a and Fig. 6b shows the plot of $\langle\bar{v}\rangle$ as a function of the friction coefficient $\gamma_{0}$ and temperature $T$ for fixed values of the drive amplitude $F_{0}$ and phase difference $\phi .\langle\bar{v}\rangle$ peaks for an optimum value of the friction coefficient $\gamma_{0}$ and temperature $T$.

When the friction in the system is very low, the cause of asymmetry in the inhomogeneous 


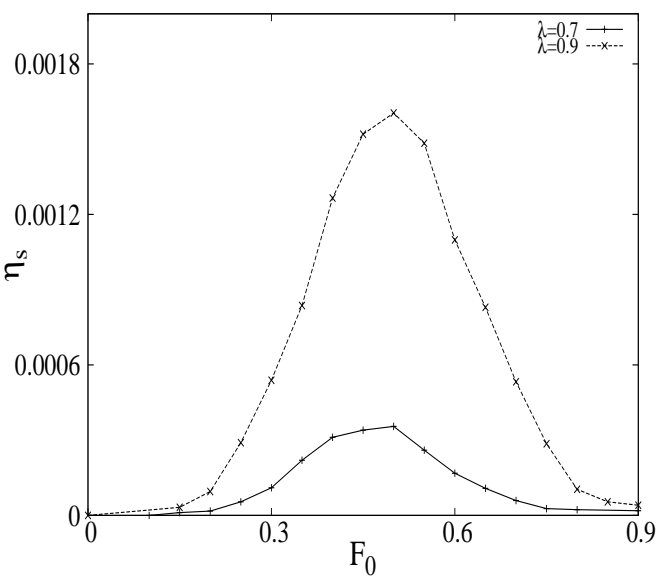

(a)

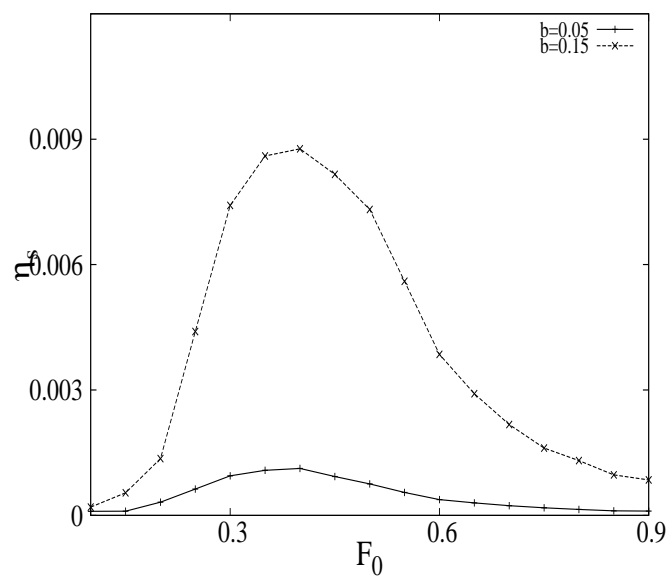

(b)

FIG. 5: Plot of Stoke's efficiency $\eta_{s}$ versus $F_{0}$ for different values of $\lambda$ (a) and $b$ (b) for $\gamma_{0}=0.12$, $\tau=1000, \phi=0.35$ and $T=0.4$.

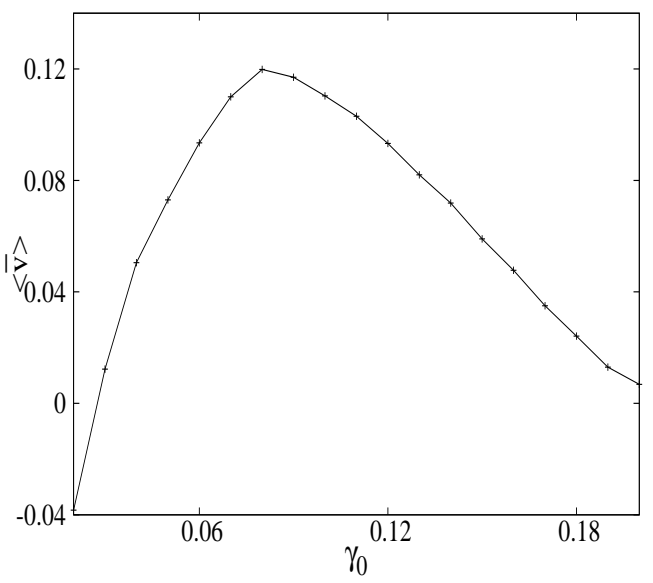

(a)

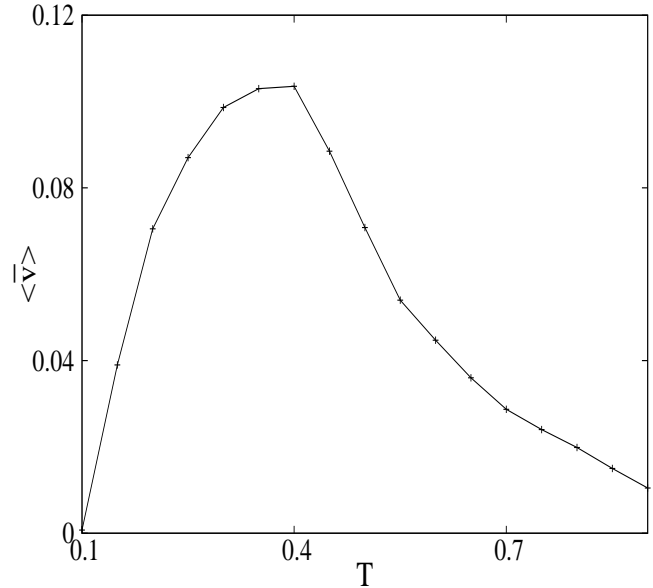

(b)

FIG. 6: Plot of $\langle\bar{v}\rangle$ versus friction coefficient $\gamma_{0}(\mathrm{a})$ and versus temperature $T(\mathrm{~b})$ for $\lambda=0.9$, $F_{0}=0.5, \tau=1000, \phi=0.35$ and $T=0.4$.

system is very feeble. As a result of which the difference between the particle hoppings to either side of a potential well will not be high which results in low particle current. As the friction is increased, the particle sees more asymmetry leading to an increase in current with the friction coefficient, ofcourse other parameters remaining fixed. However if friction is too high again particle mobility will reduce leading to less current. This explains the peaking of particle current at an optimum value of the frction coefficient.

The observed variation of particle current with temperature also can be understood by considering the fact that at low temperatures, the particles will have less probability of 
coming out of the potential wells resluting in less current. As temperature increases, the particles starts jumping out of the wells more frequently, and asymmetry in the system giving rise to a difference in the hoppings to the right and the left of the well, giving rise to a finite current. At very high temperatures though the barrier to particle motion and hence the effect of asymmetry almost vanishes reducing the current. In between these two extremes, expectedly the particle current peaks at an optimum value of temperature.

\section{EFFECT OF BOTH INHOMOGENEITY AND ASYMMETRY IN POTEN- TIAL}

We explore the pssibility of combining features of the inhomogeneous ratchet and the asymmetric ratchet to design an underdamped ratchet model having better transport characteristics and efficiency of transport. For this, a small asymmetry is added to the underlying periodic potential (finite value of $b$ ) of the inhomogeneous ratchet. Using this combination ratchet, we than calculate the various parameters characterising the transport.

In Fig. 7 the plots of the various parameters are given for the combination ratchet. It can be clearly seen that for the particular values considered $(\lambda=0.7, b=0.05)$, the average particle velocity for the combination ratchet is substantially higher than that for the individual ratchets (Fig. 7a). Interstingly, the value of the diffusion coefficient $D_{\text {eff }}$ remains almost unchanged (Fig. 7b). Thus with the combination ratchet, higher particle current is obtained without compromising on the quality of transport. This is also reflected from a measure of the Peclet number (Fig. 7c). Also the efficiency of the combination ratchet is comparatively much higher than that for the individual asymmetric ratchet and the inhomogeneous ratchet (Fig. $7 \mathrm{~d}$ ).

The combination ratchet model, which combines essential features of the inhomogeneous ratchet and the asymmetric ratchet therefore provides a new ratchet model in the underdamped regime with better transport characteristics and higher efficiency.

\section{CONCLUSION}

Study of ratchets has been an active field of research over the past few decades particulary because of its practical utility in explaining different physical and biological systems. 


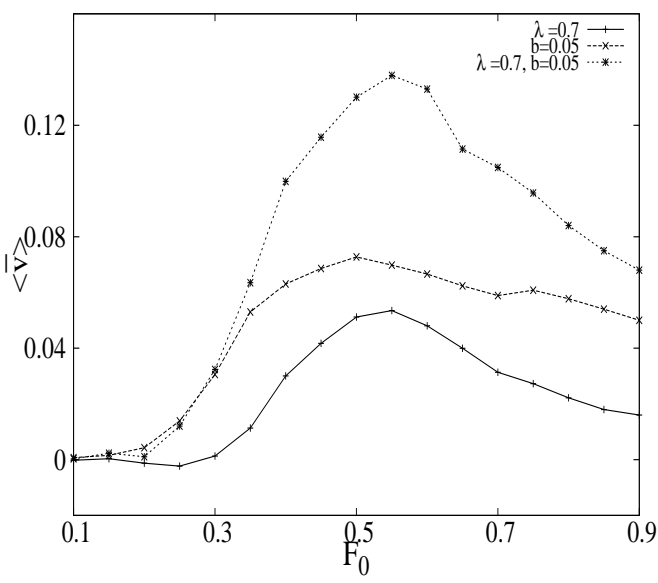

(a)

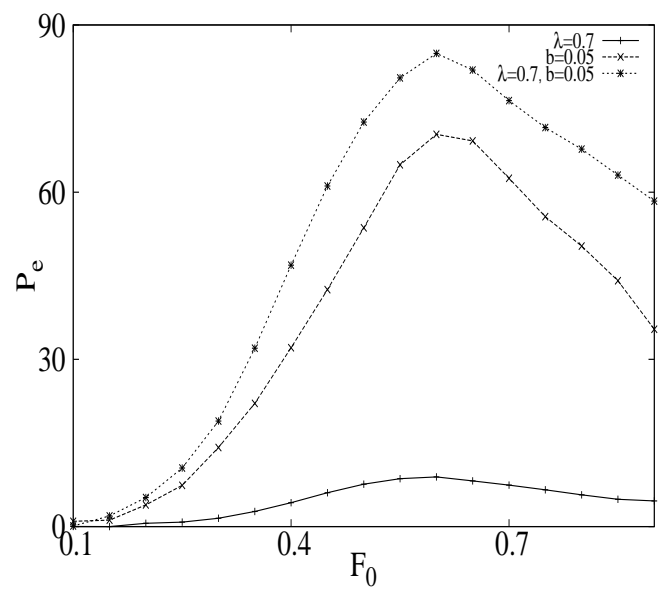

(c)

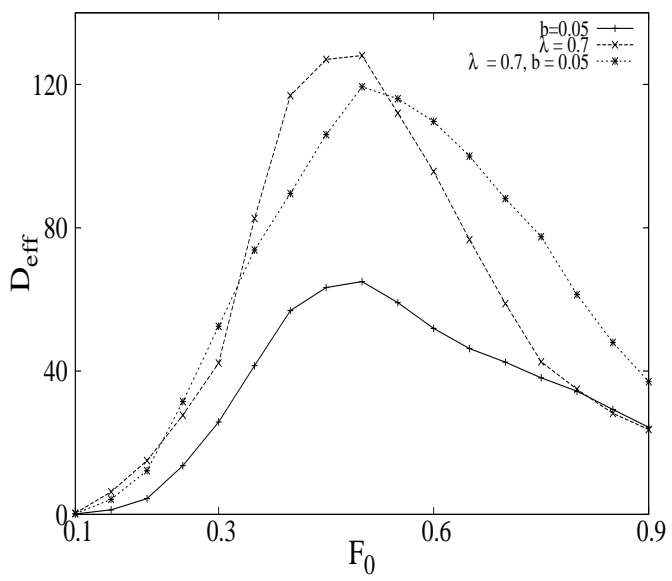

(b)

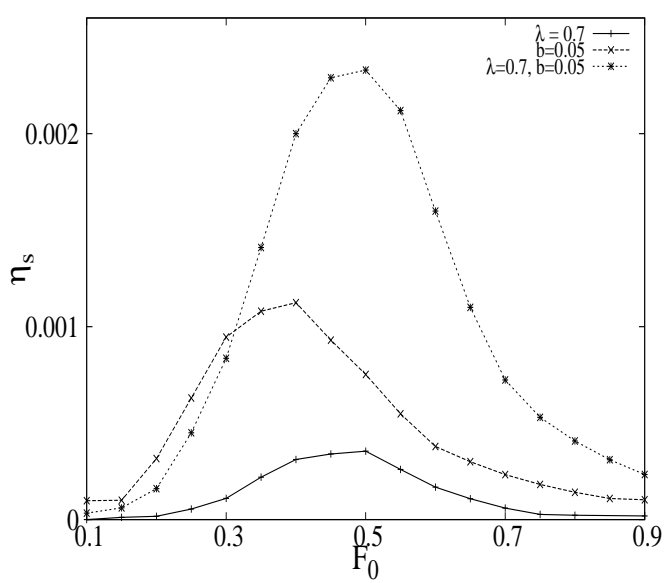

(d)

FIG. 7: Plot of the different parameters for the combination ratchet in comparision to the inhomogeneous ratchet and the asymmetric ratchet as a function of $F_{0} ;\langle\bar{v}\rangle(\mathrm{a}), D_{\text {eff }}(\mathrm{b}), P_{e}(\mathrm{c})$ and $\eta_{s}$ (d); $\tau=1000, \phi=0.35$ and $T=0.4$.

Different models of ratchets have been proposed and studied. We have studied underdamped particle transport in periodic potentials. This finds many analogies in naturally occuring systems as already discussed in the introduction. Primary focus was to study transport in an inhomogeneous ratchet with a symmetric and periodic potential but with a space dependent friction coefficient. The performance of the inhomogeneous ratchet as a function of the different parameters is studied. The transport characteristics are dependent on the various parameters governing the system. The performance of this ratchet is compared with that of the asymmetric ratchet with an asymmetric periodic potential but a homogeneous friction coefficient. It is observed that though inhomogeneity is a feeble cause of asymmetry in the system, substantial ratchet current can be obtained with the model inhomogeneous ratchet. 
We propose a new scheme of a ratchet model combining both inhomogeneity in the system and asymmetry in the underlying potential. This combination ratchet model has comparatively much better performance characteristics in the underdamped regime.

Also, the performance characteristics of the ratchet model being sensitively dependent on the choice of the various parameters governing the system, a careful choice of the different parameters becomes a necessity to optimise the working of the ratchet.

\section{ACKNOWLEDGEMENTS}

The author acknowledges financial support received from University Grants Commission (UGC), Government of India under Project No. F.5-152/2012-13/MRP/NERO/565 for carrying out this work

[1] F. Julicher, A. Ajdari and L. Prost, Rev. Mod. Phys. 69, 1269 (1997); R. D. Astumian, Scientific American 285, 56 (2001).

[2] Special issues on "Ratchets and Brownian motors: Basics, Experiments and applications", Ed. H. Linke, Appl. Phys. A 75(2), 167 (2002).

[3] R. D .Astumian and P. Hanggi, Phys. Today 55, 33 (2002).

[4] M. C. Mahato and A. M. Jayannavar, Resonance 8 (7), 33 (2003); ibid M. C. Mahato, and A. M. Jayannavar, Resonance 8 (9), 4 (2003).

[5] P. Reimann, Phys. Rep. 361, 57 (2002).

[6] J. Maddox, Nature 369, 271 (1994); ibid 369, 181 (1994).

[7] L. Gammaitoni, P. Hanggi, P. Jung, F. Marchesoni, Rev. Mod. Phys. 70, 223 (1988).

[8] R.D. Astumian, M. Bier, Phys. Rev. Lett. 72, 1766 (1994).

[9] R. Bartussek, P. Hänggi, J. G. Kissner, Europhys. Lett. 28, 459 (1994).

[10] M. O. Magnasco, Phys. Rev. Lett. 71, 1477 (1993).

[11] J. Rousselet, L. Salone, A. Ajdari, J. Prost, Nature 370, 446 (1994).

[12] C. R. Doering, W. Horsthemke, and J. Riordan, Phys. Rev. Lett. 72, 2984 (1994).

[13] M. M. Millonas, and M. I. Dykman, Phys. Lett. A. 185, 65 (1994).

[14] J. A. Spudich, Nature 372, 515 (1994).

[15] P. Hanggi and F. Marchesoni, Rev. Mod. Phys. 81, 387 (2009).

[16] Z. Siwy and A. Fulinski, Phys. Rev. Lett. 89, 198103 (2002); ibid Am. J. Phys. 72, 567 (2004); 
Z. Siwy, I. D. Kosinska, A. Fulinsky, and C. R. Martin, Phys. Rev. Lett. 94, 048102 (2005).

[17] S. Savelev, F. Nori, Nature Mater. 1, 179 (2002).

[18] P. Hanggi and F. Marchesoni, Rev. Mod. Phys. 81, 387 (2009).

[19] M. C. Mahato, T. P. Pareek and A. M. Jayannavar, Int. J. of Mod. Phys. B10, 3857(1996).

[20] A. M. Jayannavar, Phy. Rev. E. 53, 2957 (1996).

[21] D. Dan, M. C. Mahato and A. M. Jayannavar, Phys. Rev. E 60, 6421 (1999).

[22] M. C. Mahato and A. M. Jayannavar, Phys. Rev. E 63, 056307 (2001).

[23] R. Krishnan, M. C. Mahato and A. M. Jayannavar, Phys. Rev. E 70, 021102 (2004).

[24] D. Dan, A. M. Jayannavar and M. C. Mahato, Int. J. of Mod. Phys. B14, 1585(2000).

[25] M. C. Mahato and A. M. Jayannavar, Physica A 318, 154 (2003).

[26] S. Saikia and M. C. Mahato, Physica A, 389, 4052 (2010).

[27] S. Saikia and M. C. Mahato, Jour. of Phys. Cond. Mat., 21, 175409 (2009).

[28] W. L. Reenbohn and M. C. Mahato, Phys. Rev. E 91, 052151 (2015).

[29] W. L. Reenbohn and M. C. Mahato, Jour. of Stat. Mech.:Theory and Exp. Vol 2009, March 2009.

[30] W. L. Reenbohn et.al., Pramana Jour. of Phys., Vol 71, No. 2, 297 - 306 (2008).

[31] D. Kharkongor, W. L. Reenbohn and M. C. Mahato, arXiv:1607.0112v1, [cond-mat-stat-mech], 5 Jul 2016.

[32] S. Saikia and M. C. Mahato, Phys. Rev. E, 80, 082102 (2009)

[33] S. Saikia, A. M, Jayannavar and M. C. Mahato, Phs. Rev. E 83, 061121 (2011).

[34] R. Landauer, J. Stat. Phys. 53, 233 (1988).

[35] M. Buttiker, Z. Phys. B 68, 161 (1987).

[36] Ya. M. Blanter and M. Büttiker, Phys. Rev. Lett. 81, 4040 (1988).

[37] R. Benjamin and R. Kawai, Phys. Rev. E 77, 051132 (2008).

[38] R. H. Luchsinger, Phys. Rev. E. 62, 272 (2000).

[39] L. P. Faucheux and A. J. Libchaber, Phys. Rev. E 49, 5158 (1994).

[40] H. Brenner, Chem. Eng. Sci. 16, 242 (1962).

[41] C. M. Falco, Am. J. Phys. 44, 733 (1976); A. Barone and G. Patterno Physics and applications of the Josephson effect, John Wiley, New York, 1982.

[42] G. Wahnstrom, Surf. Sci. 159, 311 (1985).

[43] S. Flach, O. Yevtushenko, and Y. Zolotaryuk, Phys. Rev. Lett. 84, 2358 (2000). 
[44] P. Jung, J. G. Kissner and P. Hänggi, Phys. Rev. Lett. 76, 3436 (1996).

[45] L. Machura, M. Kostur, F. Marchesoni, P. Talkner, P. Hänggi and J. Luczka, J. Phys. Cond. Matter 17, S3741 (2005); L. Machura, M. Kostur, P. Talkner, J. Luczka, F. Marchesoni and P. Hänggi, Phys. Rev. E 70, 061105 (2004).

[46] K. Lindenberg, J. M. Sancho, A. M. Lacasta and I. M. Sokolov, Phys. Rev. Lett. 98, 020602 (2007).

[47] C. Constantini and F. Marchesoni, Europhys. Lett. 48, 491 (1999).

[48] B. Lindner et. al. Phys. Rev. E 59, 1417 (1999).

[49] P. Reimann, R. Bartussek, R. Haubler, and P. Hanggi, Phys. Lett. A 215, 26 (1996).

[50] E. Pollak, J. Bader, B. J. Berne and P. Talkner, Phys. Rev. Lett. 70, 3299 (1993); M. Borromeo and F. Marchesoni, Phys. Rev. Lett. 84, 203 (2000); O. M. Braun and R. Ferrando, Phys. Rev. E 65, 061107 (2002).

[51] M. Borromeo, G. Constantini and F. Marchesoni, Phys. Rev. Lett. 82, 2820 (1999); G. Constantini and F. Marchesoni, Europhys. Lett, 48, 491 (2000).

[52] O. M. Braun, A. R. Bishop and J. Roder, Phys. Rev. Lett. 79, 3692 (1997); C. Cattuto and F. Marchesoni, Phys. Rev. Lett. 79, 5070, (1997).

[53] E. Ben-Jacob, D. J. Bergman, B. J. Matkowsky and Z. Schuss, Phys. Rev. A, 26, 2805 (1982).

[54] H. Risken, The Fokker-Planck Equation (Springer, Berlin, 1984).

[55] B. Lindner and I. M. Sokolov, Phy. Rev. E, 93, 042106 (2016).

[56] O. M. Braun and R. Ferrando, Phys. Rev. E, 65(6), 061107 (2002).

[57] M. Borromeo, G. Constantini and F. Marchesoni Phys. Rev. E, 65, 041110 (2002).

[58] L . Machura et. al., Phys. Rev. E, 70, 061105 (2004).

[59] P. Jung, J. G. Kissner and P. Hanggi, Phys. Rev. Lett., 76, 18, 3136 (1996).

[60] F. Marchesoni, S. Savalev and F. Nori, Phys. Rev. E, 73, 021102 (2006).

[61] H. Linke, M. T. Downton, and M. J. Zuckermann, Chaos 026111 (2005).

[62] L. D. Landau and E. M. Lifshitz, Fluid Mechanics, (Pergamon Press, New York, 1959) p.203.

[63] K. Sekimoto, J. Phys. Soc. Jpn. 66, 1234 (1997).

[64] D. Suzuki and T. Munakata, Phys. Rev. E 68, 021906 (2003).

[65] R. Mannella, A Gentle Introduction to the Integration of Stochastic Differential Equations, in Stochastic Processes in Physics, Chemistry, and Biology, edited by J. A. Freund and T. P oschel, Lecture Notes in Physics Vol. 557 (Springer, Berlin, 2000), p. 353. 